\title{
Genotype by environment interaction for first-lactation female fertility traits in UK dairy cattle
}

\author{
E. Strandberg, ${ }^{* 1}$ S. Brotherstone, † E. Wall, $\ddagger$ and M. P. Coffey $\ddagger$ \\ *Department of Animal Breeding and Genetics, Swedish University of Agricultural Sciences, PO Box 7023, S-75007 Uppsala, Sweden \\ †Institute of Evolutionary Biology, University of Edinburgh, West Mains Road, Edinburgh, United Kingdom \\ ‡Sustainable Livestock Systems Group, Scottish Agricultural College, Bush Estate, Penicuik, Midlothian, EH26 OPH, United Kingdom
}

\section{ABSTRACT}

The objective of this study was to examine whether there was genotype by environment interaction $(\mathrm{G} \times \mathrm{E})$ for female fertility traits for various environmental descriptors used in the United Kingdom. Records on days to first service (DFS), nonreturn rate at $56 \mathrm{~d}$ (NR56), calving interval (CI), and number of inseminations (NINS) on approximately 200,000 first-lactation Holstein cows in 3,192 herds and from 1,147 sires were analyzed using both random regression reaction norm models and multiple-trait models. The environmental descriptors were herd averages of DFS, CI, and NR56, and herd production and intensity indices, the latter based on 305-d milk, fat, and protein yields, age at first calving, temperature, and rainfall. No $\mathrm{G} \times \mathrm{E}$ was found for NR56 and NINS. There was an indication of $\mathrm{G} \times \mathrm{E}$ for DFS and CI with respect to the herd average of that trait, but only from the reaction norm model. Genotype by environment interaction existed for DFS and CI with respect to both production and intensity indexes - genetic correlations between top and bottom quartiles ranged from 0.73 to 0.84 , with similar results from both models, indicating reranking of bulls. Part of this $\mathrm{G} \times \mathrm{E}$ might be explained by low production and intensity herds also having more concentrated spring calving.

Key words: genotype by environment interaction, female fertility, dairy cattle

\section{INTRODUCTION}

Dairy cow fertility is becoming increasingly important throughout the world. More countries are starting to do genetic evaluations for female fertility, and in April 2008, 18 countries participated in the international genetic evaluation for female fertility (www-interbull. slu.se).

Received October 28, 2008.

Accepted March 21, 2009.

${ }^{1}$ Corresponding author: Erling.Strandberg@hgen.slu.se
Although globalization increases the environmental range that the daughters of sires encounter, there is also a tendency toward more diverse production environments within country. In Europe there is a political will that a larger proportion of the land should be organically farmed, which in general would mean a lower intensity of production, more grazing, and restrictions on feedstuffs and pharmaceutical treatments for those farms.

With an increasing range of environments, the potential importance of genotype by environment interaction $(\mathbf{G} \times \mathbf{E})$ increases. On a global scale, it can have large consequences for the selection of bulls, but it may also be important on a national scale, where individual farmers could choose bulls that are more appropriate for their type of production system.

Results from international bull evaluations (Interbull) have shown rather low genetic correlations between some countries, especially between countries with predominantly grazing systems (e.g., New Zealand) and other industrialized countries (e.g., Europe, United States). With correlations as low as 0.6 for fertility traits, there would be substantial reranking of bulls.

There are relatively few studies on $\mathrm{G} \times \mathrm{E}$ for female fertility within a country. Generally, these studies have found very little evidence for $\mathrm{G} \times \mathrm{E}$ and most genetic correlations between environments have not been significantly different from unity (Kolmodin et al., 2002; Boettcher et al., 2003; Kearney et al., 2004; Calus et al., 2005; Windig et al., 2006). Studies on $\mathrm{G} \times \mathrm{E}$ for fertility traits in relation to UK environmental conditions have not been carried out. The objective of this study was to investigate whether there is $\mathrm{G} \times \mathrm{E}$ for female fertility traits for various UK environmental descriptors.

\section{MATERIALS AND METHODS}

\section{Data}

Milk production data consisted of 305-d records for lactation 1 to lactation 5 for milk $(\mathbf{M})$, fat $(\mathbf{F})$, and protein (P) yields from calvings between 1998 and 
Table 1. Number of observations, means, SD, minimum and maximum (Min-Max) values for various fertility traits in first-lactation cows (data set C)

\begin{tabular}{lcccc}
\hline Trait $^{1}$ & $\mathrm{n}$ & Mean & SD & Min-Max \\
\hline DFS & 226,325 & 86.4 & 33.1 & $3-200$ \\
CI & 199,469 & 400 & 58.3 & $301-599$ \\
NR56 & 225,855 & 0.674 & 0.469 & $0-1$ \\
NINS & 193,373 & 1.73 & 1.07 & $1-10$ \\
\hline
\end{tabular}

${ }^{1} \mathrm{DFS}=$ days to first service; $\mathrm{CI}=$ calving interval; NR56 = nonreturn rate at $56 \mathrm{~d}$; NINS $=$ number of inseminations needed for pregnancy.

2004. These data were collected from 2 major UK milk recording organizations. The fertility traits were those available in the database used for national genetic evaluation: days to first service (DFS), nonreturn rate at $56 \mathrm{~d}$ (NR56), calving interval (CI), and number of inseminations needed for a subsequent calving (NINS), all pertaining to first lactation. Of these, NR56 and CI are used in the current UK fertility index. From calving records, age at first calving (AGE1C) was also determined and an average age at first calving calculated for each herd during the years 1998-2004 $(\overline{A G E 1 C})$. Using the farm postal codes recorded by the milk recording organizations, the closest meteorological station was identified and the average temperature $(\bar{T})$ and rainfall $(\bar{R})$ for each herd were obtained, based on monthly averages during the years 1999 to 2003 (Haskell et al., 2007).

After deleting first-lactation records where AGE1C was $<18$ mo or $>40 \mathrm{mo}$ and deleting herds with fewer than 5 first-lactation cows, 1,221,475 production records from 3,886 herds remained (data set $\mathrm{A}$ ). Of these, 280,798 cows had some first-lactation fertility records (data set B). These were from 7,682 sires in 3,739 herds. From data set B, progeny of sires with at least 30 daughters were extracted. From these records, cows in herds with $<10$ observations were deleted. This left a data set (C), which contained 232,437 daughter records in 3,192 herds, from 1,147 sires. The number of observations and descriptive statistics of the fertility traits are shown in Table 1. This data set was used for the genetic analyses described below.

\section{Environmental Descriptors}

Five environmental descriptors were used: an intensity index, a production index, and 3 fertility descriptors. The intensity index (Eq. [1]) was developed in Haskell et al. (2007) as that linear combination of variables available for all herds that had the highest correlation with herd environmental variables collected from a questionnaire on 778 participating herds. The intensity index for each herd was defined as:

$$
\begin{aligned}
I_{\text {Int }}= & -1.0 \times \bar{T}-0.092 \times \bar{R}-0.296 \times \overline{A G E 1 C} \\
& +0.005 \hat{M}+0.175 \times \hat{P}-0.09 \times \hat{F},
\end{aligned}
$$

where $\bar{T}$ is the average temperature $\left({ }^{\circ} \mathrm{C}\right)$ and $\bar{R}$ is the average rainfall $(\mathrm{mm}) ; \hat{M}, \hat{P}$, and $\hat{F}$ were herd solutions from the following model:

$$
y_{i j k l}=\mu+l a c_{j}+\beta_{j} A G E_{j}+y m_{k}+h e r d_{l}+e_{i j k l},
$$

where $y_{i j k l}$ is the 305-d milk, fat, or protein yield of the cow, $\mu$ is the overall mean, $l a c_{j}$ is the effect of the $j$ th lactation number $(j=1 \ldots 5), \beta_{j}$ is the regression coefficient of the trait on age at calving for the $j$ th lactation, $y m_{k}$ is the effect of the $k$ th year-month of calving, $h e r d_{l}$ is the effect of the lth herd, and $e_{i j k l}$ is the random residual term. All factors were fixed except the residual. Age at calving $(\boldsymbol{A} \boldsymbol{G} \boldsymbol{E})$ was expressed as within-lactation deviation from the mean age $30,44,58,72$, and 86 mo for the 5 lactations, respectively. Before being used in Eq. [1], herd solutions were adjusted to lactation-2 equivalents (i.e., $\hat{\mu}+\widehat{l a c_{2}}$ was added). This model was applied to data set $\mathrm{A}$.

Apart from the intensity index, the herd solutions from Eq. [2] for milk, fat, and protein yields were averaged and used as an environmental descriptor: a herd production index. Furthermore, herd solutions from model [2] (without effect of lactation number and with regression on AGE1C) applied to the traits DFS, CI, and NR56 (in first lactation) were used as measures of herd fertility. Herd solutions were standardized (mean zero, SD unity), as were the environmental descriptors, before being used in the models for estimation of $\mathrm{G} \times \mathrm{E}$. Means and standard deviations of the environmental descriptors before standardization are given in Table 2 . The herd variables relatively well approximated a normal distribution, but with some skewness and kurtosis. The 2 environmental indices (average of $\hat{M}, \hat{F}$, and $\hat{P}$ and the intensity index) were even closer to a normal distribution. Visually, all 4 were unimodally normally distributed.

\section{Models for Estimating GXE}

We used several models to estimate variation in environmental sensitivity among sires, which is a prerequisite for $\mathrm{G} \times \mathrm{E}$. The traits studied were DFS, NR56, CI, and NINS, all measured in first lactation.

Reaction Norm Models. The first type of model used random regressions to estimate reaction norms $(\mathbf{R N})$ for sires. The base model was a sire model including a sire by herd interaction: 
Table 2. Mean and standard deviation of environmental descriptor variables before standardization (data set $\mathrm{C})^{1}$

\begin{tabular}{lcc}
\hline Trait $^{2}$ & Mean & SD \\
\hline Herd effect, 305-d milk yield, $\hat{M}$ & 7,234 & 1,182 \\
Herd effect, 305-d fat yield, $\hat{F}$ & 278.6 & 44.59 \\
Herd effect, 305-d protein yield, $\hat{P}$ & 236.0 & 38.22 \\
Herd effect DFS & 88.94 & 13.48 \\
Herd effect CI & 399.6 & 17.69 \\
Herd effect NR56 & 0.701 & 0.115 \\
Intensity index, $I_{\text {Int }}$ & 26.17 & 9.47 \\
\hline
\end{tabular}

${ }^{1}$ Herd effects for milk production traits are expressed on a lactation-2 basis.

${ }^{2} \mathrm{DFS}=$ days to first service; $\mathrm{CI}=$ calving interval; NR56 $=$ nonreturn rate at $56 \mathrm{~d}$.

$$
y_{i j k l}=\mu+A G E 1 C_{i}+y m_{j}+h_{k}+s h_{k l}+s_{0 l}+e_{i j k l},
$$

where $A G E 1 C_{i}$ is the fixed effect of age at first calving $(18,19, \ldots 40), y m_{j}$ is the fixed effect of year-month of first calving, $h_{k}$ is the fixed effect of herd $k, s_{0 l}$ is the random sire effect for sire $l$, and $s h_{k l}$ is the random sire by herd interaction. Model [3] was complemented with a linear random regression of the trait on the environmental descriptor for each sire (reaction norm):

$$
\begin{gathered}
y_{i j k l}=\mu+A G E 1 C_{i}+y m_{j}+h_{k} \\
+s h_{k l}+s_{0 l}+s_{1 l} X+e_{i j k l},
\end{gathered}
$$

where $s_{0 l}$ and $s_{1 l}$ are the level and slope of the reaction norm, respectively. Model [4] contains the variable $X$, the environmental descriptor used in the particular analysis. We did not include a fixed (average) reaction norm, because this was accounted for by the herd effect. We tested the linear RN model [4] against model [3] using a likelihood ratio test. If model [4] was better, we also tested whether the variance component for the linear term was significantly different from zero. We considered there to be significant $\mathrm{G} \times \mathrm{E}$ if both these criteria were met. If the random linear reaction norm was significant, we also tested a quadratic reaction norm model (against model [4]):

$$
\begin{gathered}
y_{i j k l}=\mu+A G E 1 C_{i}+y m_{j}+h_{k} \\
+s h_{k l}+s_{0 l}+s_{1 l} X+s_{2 l} X^{2}+e_{i j k l} .
\end{gathered}
$$

All models were analyzed using an average-information REML approach in the statistical program DMU (Madsen and Jensen, 2000). The variance-covariance structure for model [5] was:

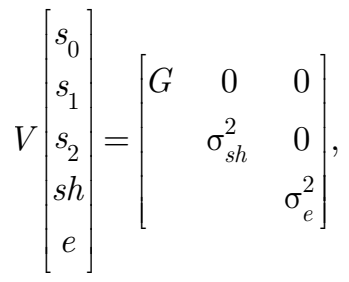

where

$$
G=\left[\begin{array}{ccc}
\sigma_{s 0}^{2} & \sigma_{s 01} & \sigma_{s 02} \\
& \sigma_{s 1}^{2} & \sigma_{s 12} \\
& & \sigma_{s 2}^{2}
\end{array}\right] .
$$

For the models [4] or [3], the last 1 or 2 rows/columns were deleted.

Even though the trait NR56 is of binary nature, we analyzed it with a linear model rather than with a threshold model. It has been shown previously that the linear model works well in a sire model setting to estimate genetic parameters that are unbiased if transformed to the underlying scale (Mantysaari et al., 1991). The possible heterogeneity of residual terms was checked by calculating the variance of the residual terms from the model within 10 intervals of the environmental scale used in the model, where each interval consisted of approximately equal number of herds. These calculated residual variances were checked visually for any trend across environments.

The sire $\times$ herd effect represents residual effects unexplained by the reaction norm for each sire. Two herds with the same $X$-value in the regression for the reaction norm could have different sire $\times$ herd terms. Having this term improved the fit of the model (not shown). As explained in Haskell et al. (2007), retaining this term is also important in preserving the correct variancecovariance structure in the mixed model. Omitting it would create a heterogeneous residual variance and possibly biased tests of other fixed and random effects in the model.

Multiple-Trait Models. Apart from the random regression models, a multiple trait (MT) analysis was carried out where the environments were categorized into low or high (lowest and highest $25 \%$ of the observations, respectively). The average values of the environmental descriptors for the 2 quartiles are shown in Table 3. The trait values were then considered as 2 separate traits and analyzed using model [3] in a bivariate setting:

$$
y_{i j k l t}=\mu+A G E 1 C_{i t}+y m_{j t}+h_{k t}+s h_{k l t}+s_{0 l t}+e_{i j k l t},
$$


Table 3. Average value of the environmental index for the lowest and highest quartile of observations in data set $\mathrm{C}$, used in the multipletrait analysis

\begin{tabular}{lcc}
\hline & \multicolumn{2}{c}{ Quartile values } \\
\cline { 2 - 3 } Environmental scale $^{1}$ & Low & High \\
\hline DFS & -1.2 & 1.2 \\
CI & -1.1 & 1.2 \\
NR56 & -1.4 & 1.0 \\
Production & -1.0 & 1.5 \\
Intensity & -1.0 & 1.5 \\
\hline
\end{tabular}

${ }^{1}$ DFS $=$ days to first service; $\mathrm{CI}=$ calving interval; NR56 = nonreturn rate at $56 \mathrm{~d}$.

where index $t$ indicates the trait. The assumed variancecovariance matrix of the random effects was

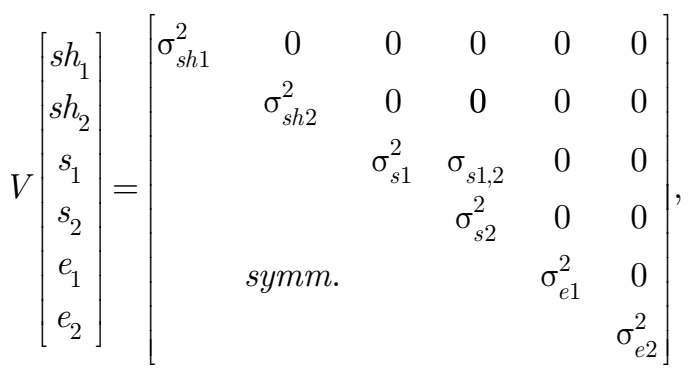

where the subscripts 1 and 2 indicate the 2 traits, respectively. A genetic correlation between the 2 traits (environments) significantly different from unity was used as an indication of $\mathrm{G} \times \mathrm{E}$.

\section{RESULTS AND DISCUSSION}

Phenotypically, DFS was quite strongly correlated to CI, which was expected because DFS is a component of calving interval. Similarly, NINS was strongly correlated both to NR56 and CI. Days to first service was only weakly correlated to NR56 (and NINS), indicating that these traits measure different aspects of fertility (Table 4).

Herds with longer DFS had longer CI, but also somewhat better pregnancy rates (Table 4). This might be interpreted as that herds that achieve good fertility results (high NR56 and few NINS) do so partly by delaying first service. The correlation structure between the herd averages made it impractical to define a single measurement of herd fertility: DFS and CI are both influenced by the voluntary waiting period and the cow's ability to start cycling after calving, and therefore positively correlated, but NR56 was positively correlated with DFS and uncorrelated with CI. Similarly, the correlations of DFS and CI with NINS had different signs. Calus et al.
(2005) found a similar relationship - herds with longer DFS had fewer NINS but longer CI.

Based on these results we decided to use 3 herd fertility environmental scales (DFS, CI, and NR56) but to exclude NINS because it was highly correlated with NR56, and because the last insemination was sometimes created in the editing procedure before national genetic evaluation, when the time between the last reported insemination and an actual calving was unrealistically long. Furthermore, herd-average DFS and, to a somewhat lesser extent, CI are more a decision of the farmer, whereas NR56 is more a measure of the ability of the farmer to detect heat and inseminate at the correct time, apart from having a general environment conducive to cows becoming and remaining pregnant.

\section{Results from RN Models}

The parameter estimates from the sire model [3] and from the linear RN model [4] where this fitted better than [3] are shown in Table 5. For DFS, the linear RN model was better than the ordinary sire model for all environmental scales. For CI and NINS, model [4] was better for some of the environments, whereas for NR56, model [4] was better only when the scale was herdaverage NR56. This trait also showed almost no genetic variation. However, closer examination of the variance component for the slope showed that it was only significantly different from zero in some of the cases where model [4] fitted better than model [3] (Table 5). To summarize, DFS and CI showed $\mathrm{G} \times \mathrm{E}$ with respect to the herd average of the trait itself and herd production and intensity. The only other trait that showed $\mathrm{G} \times \mathrm{E}$ was NINS with respect to herd-average NR56.

For those trait-environment combinations where the random linear reaction norm was significant, we tested a quadratic reaction norm but found it not significant.

The heritability estimates for all traits where $\mathrm{G} \times \mathrm{E}$ was found are shown in Figures 1 and 2. For DFS, heritability was relatively constant on the production and intensity scales, but was slightly higher in low-pro-

Table 4. Correlation among phenotypic observations (above diagonal) and among herd solutions (below diagonal) for fertility traits (data set C)

\begin{tabular}{lrrrr}
\hline & \multicolumn{4}{c}{ Trait $^{1}$} \\
\cline { 2 - 5 } Trait & DFS & CI & NR56 & NINS \\
\hline DFS & - & 0.50 & 0.04 & -0.07 \\
CI & 0.63 & - & -0.28 & 0.65 \\
NR56 & 0.27 & -0.02 & - & -0.64 \\
NINS & -0.23 & 0.37 & -0.69 & - \\
\hline
\end{tabular}

$\overline{{ }^{1} \mathrm{DFS}=\text { days to first service; } \mathrm{CI}=\text { calving interval; NR56 = nonreturn }}$ rate at $56 \mathrm{~d}$; NINS $=$ number of inseminations needed for pregnancy. 
Table 5. Estimates of variance components for level, slope, sire $\times$ herd interaction, and residual $\left(\hat{\sigma}_{b 0}^{2}, \hat{\sigma}_{b 1}^{2}, \hat{\sigma}_{s h}^{2}\right.$, and $\left.\hat{\sigma}_{e}^{2}\right)$, correlation between level and slope $\left(\hat{r}_{b 0, b 1}\right)$, and heritability $\left(h^{2}\right.$, in $\%$, in the average environment) from the various single-trait reaction norm (RN) models for various environmental descriptors

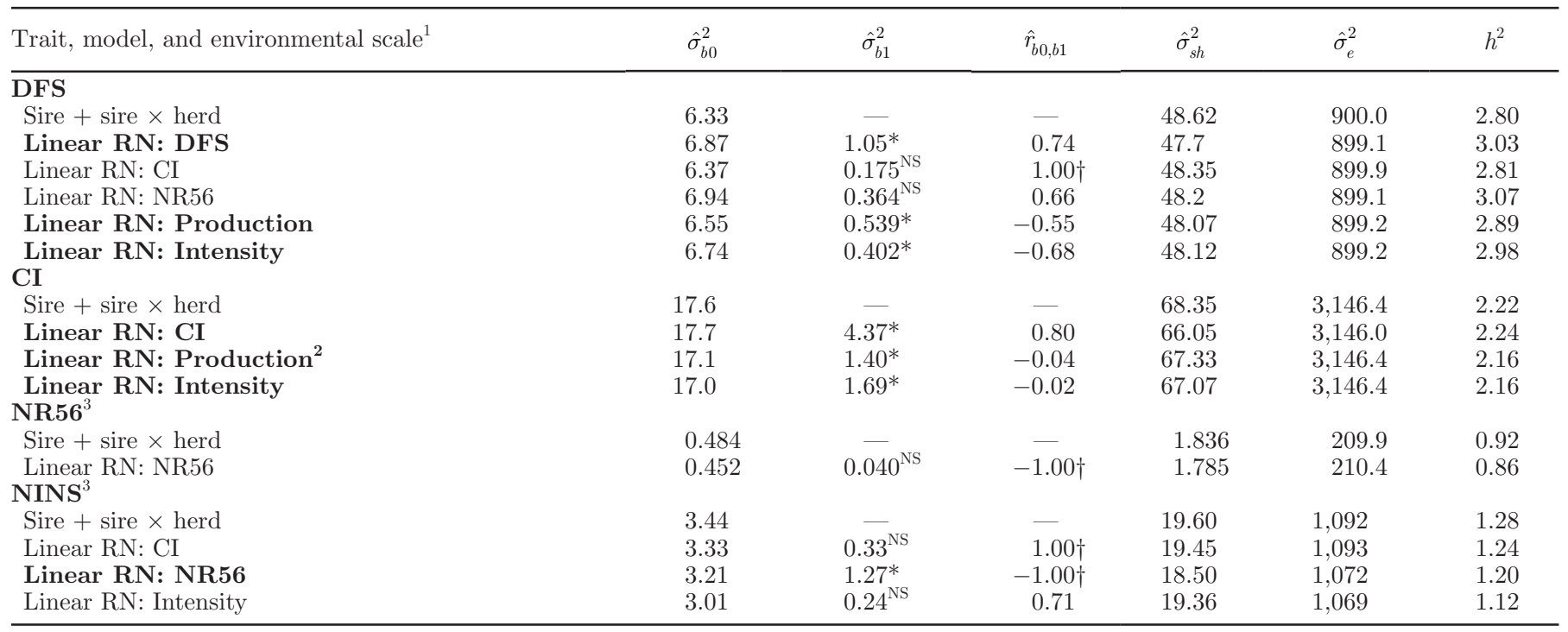

${ }^{1}$ Only significant RN models are presented and those with variance of slope significantly different from zero are shown in bold; DFS $=$ days to first service; $\mathrm{CI}=$ calving interval; NR56 = nonreturn rate at $56 \mathrm{~d}$; NINS = number of inseminations needed for pregnancy. Variances are multiplied by $10^{3} ;{ }^{*}$ and ${ }^{\mathrm{NS}}$ indicate whether the variance component for the slope is significant (5\%, one-sided test) or not.

${ }^{2}$ Model not significantly better than model without RN but variance of slopes significantly different from zero.

$\dagger$ Genetic correlation $>0.995$, rounded to 1.00 .

duction and low-intensity herds (Figure 1). Heritability increased with increasing herd DFS. A similar increase was found for the heritability of CI with respect to herd-average CI (Figure 2). Calus et al. (2005) found higher genetic variance for DFS and days to last service in herds with long calving intervals, and for days from first to last service and NINS in herds with a greater average number of inseminations. Our results are similar to results found by Kolmodin et al. (2002) for days open, where the lowest heritability was estimated in herds with short average days open. In that study the reason suggested was a "natural" lower limit of days open, which limited the phenotypic variation and thus the genetic variation. A similar reason is likely in this study, the effect of which is even more clear for the heritability of NINS, which approached zero in herds with high NR56 (Figure 2).

The largest heterogeneity of residual variance from model [4] was found for DFS with respect to the herd DFS scale, but less for the other traits and scales (not shown). However, if this heterogeneous residual variance was accounted for when calculating the heritability, it resulted in only minor changes in heritability (Figure 1 ), leading to the conclusion that for these traits, accounting for residual heterogeneity was not crucial.

The genetic correlations across the environmental range are shown in Figure 3 for the trait DFS with respect to herd-average DFS. There was a high correlation among herds with average or higher DFS values, whereas the correlation between these and low fertility herds was substantially lower.

The genetic correlation of DFS across the herd production scales is shown in Figure 4. The correlation among low and average herds was relatively high, whereas the correlation between these and high-level herds was somewhat lower. Genetic correlations with respect to herd intensity were slightly higher than those with respect to production (not shown).

The genetic correlation for CI with respect to herdaverage CI (Figure 5) followed a similar pattern to that for DFS with respect to DFS (Figure 3), albeit more extreme. Calving intervals observed in low and high CI herds were relatively weakly correlated. However, in interpreting these correlations one should remember that a herd with an average CI of $2 \mathrm{SD}$ below the average would have a CI of approximately $1 \mathrm{yr}$, and obviously very few herds achieve this.

The correlations for CI with respect to herd intensity (Figure 6) and production (not shown) were similar to each other (the former showing somewhat more $\mathrm{G} \times \mathrm{E}$ ) and to those for DFS with respect to production or intensity.

None of the RN models for NR56 showed $\mathrm{G} \times \mathrm{E}$, and genetic correlations across environments are therefore 


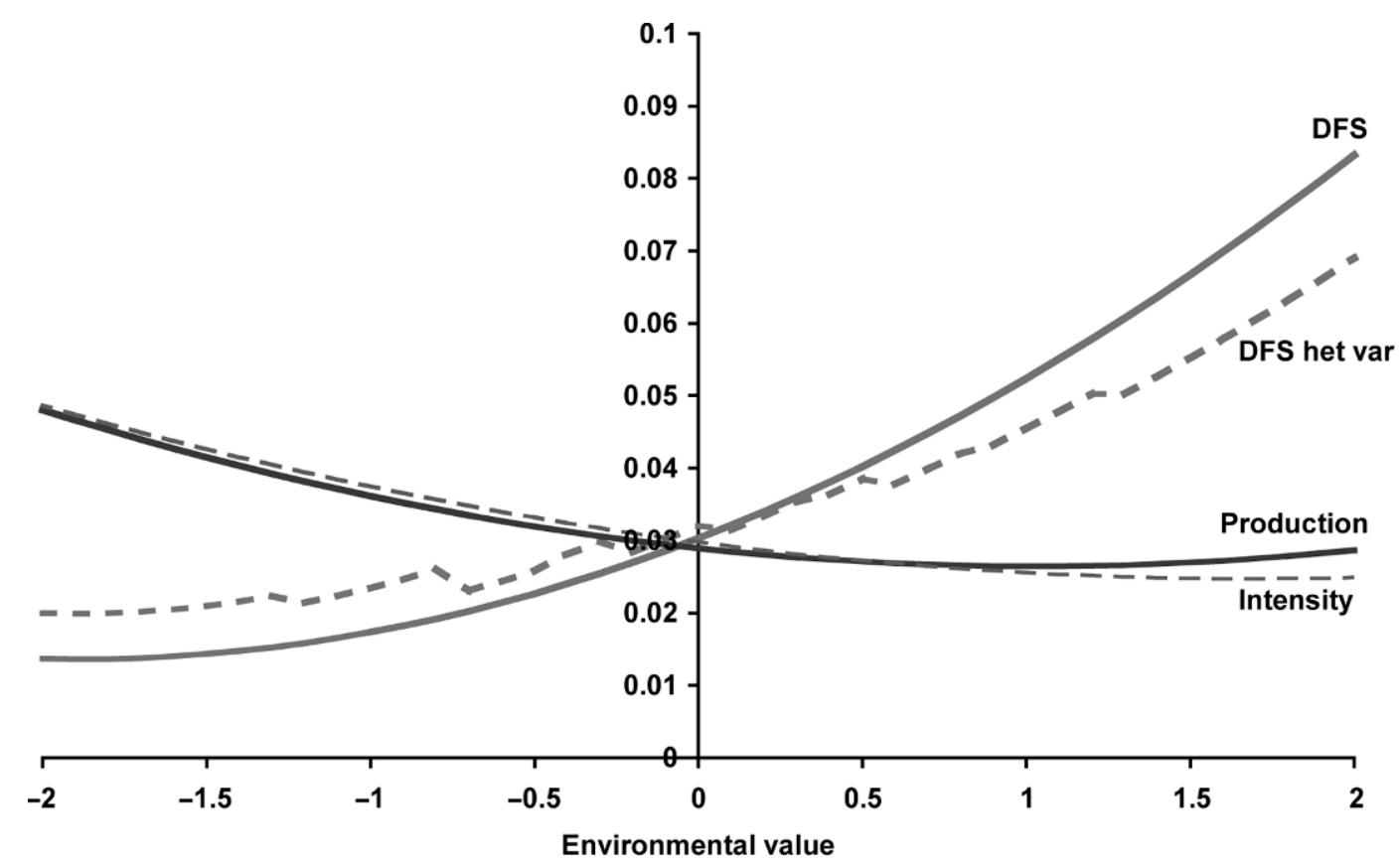

Figure 1. Heritability estimates for days to first service (DFS) as a function of various environmental descriptors (DFS, production, and intensity) and heritability when heterogeneous residual variance is accounted for (for environment DFS).

not shown. The genetic correlations for NINS with respect to herd-average NR56 (not shown) were very high across all environments except around the point where the genetic variance was close to zero (Figure 2 ), where all reaction norms converged. This should be considered an artifact of the environmental scale rather than true reranking of individuals. Another indication that the RN models did not work well for NR56 and NINS is the strong correlation between the level and the slope (Table 5).

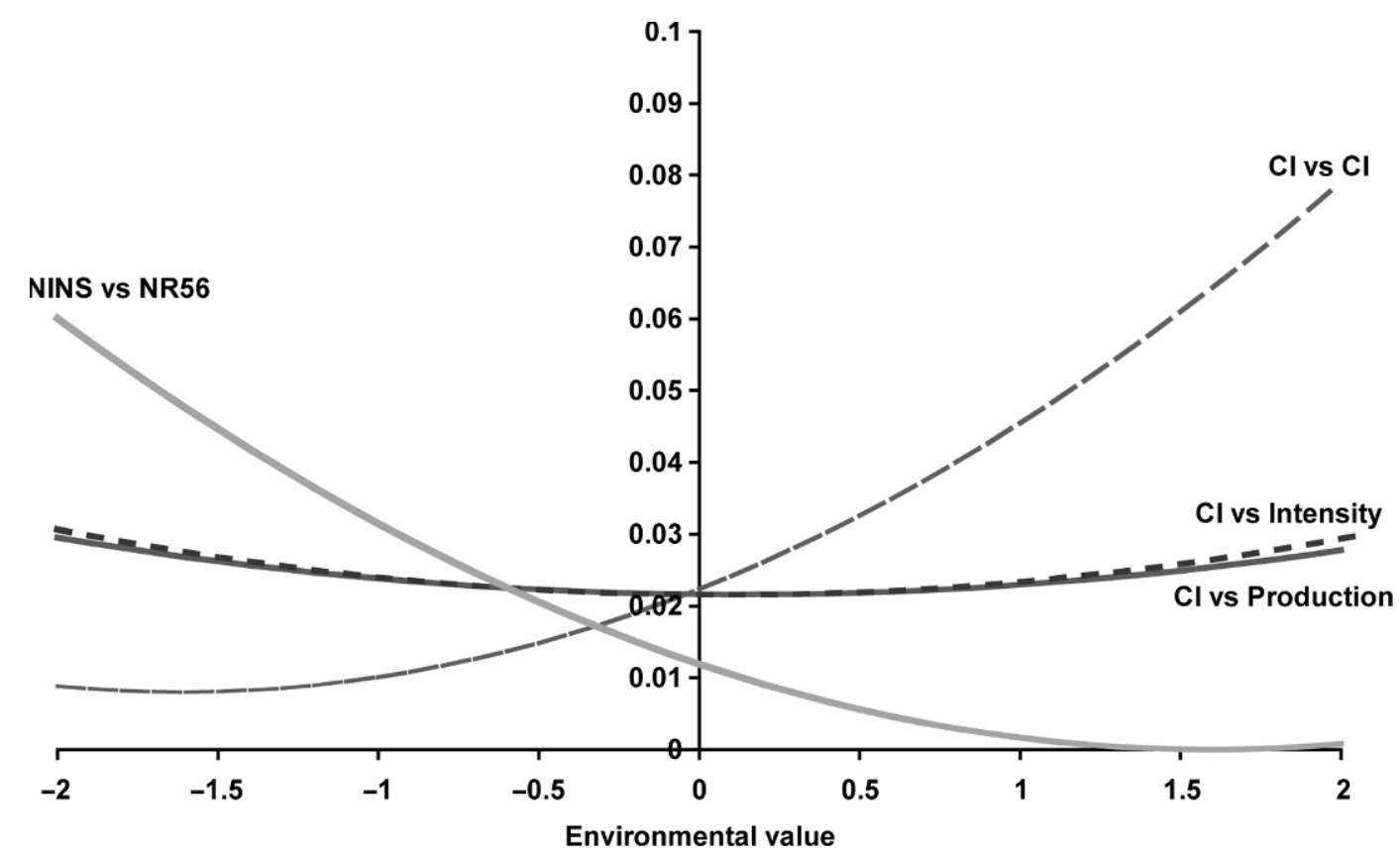

Figure 2. Heritability estimates for calving interval (CI) and number of inseminations needed for a subsequent calving (NINS) as a function of various environmental descriptors: CI, nonreturn rate at $56 \mathrm{~d}$ (NR56), production, and intensity. 


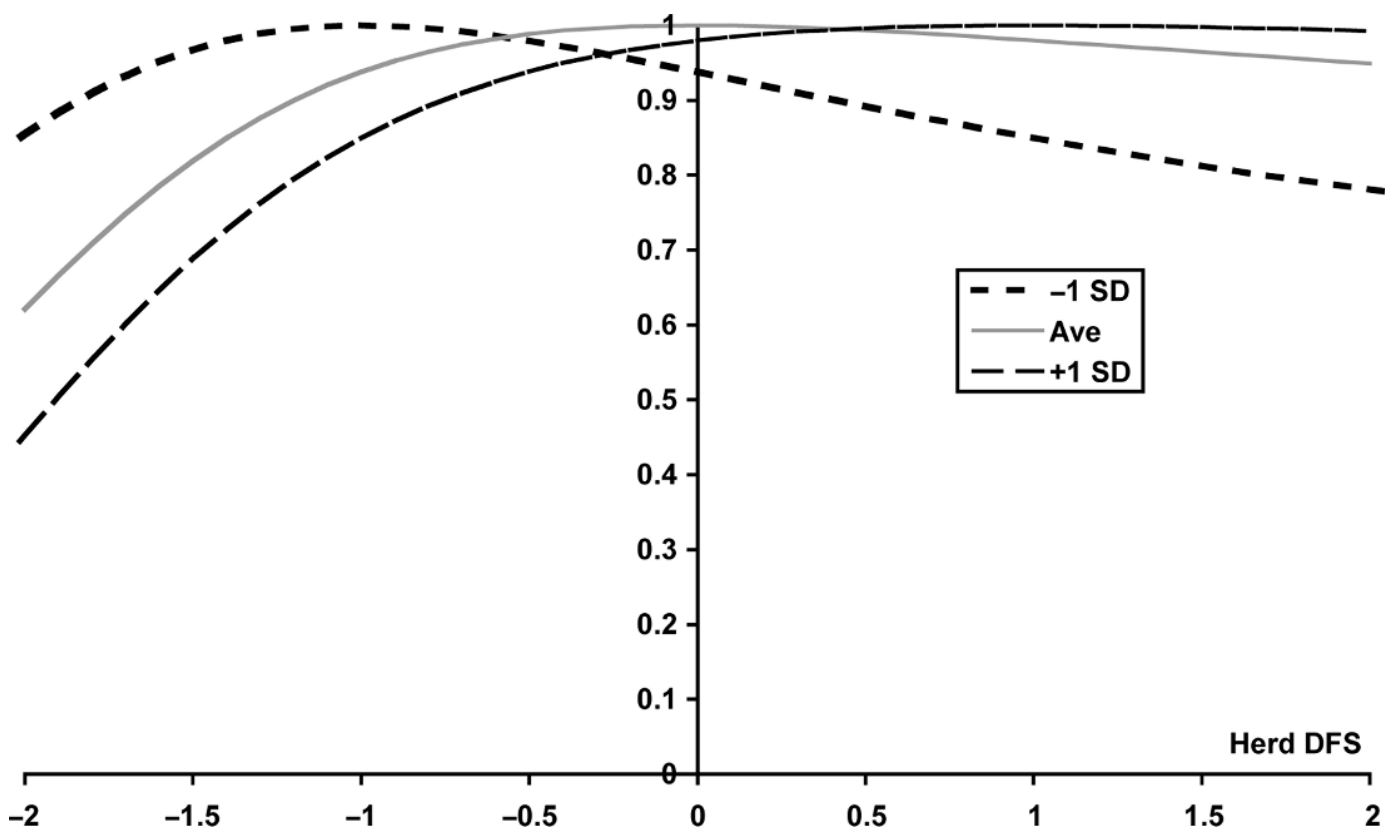

Figure 3. Genetic correlations for days to first service (DFS) across various herd DFS values. Graphs shown are between herds with environmental values of -1 , average, and $+1 \mathrm{SD}$, respectively, and all other environmental values.

In this study we used the common way to define the environmental values as the estimates of the herd effect from a separate model. It has been shown that a better approach is to estimate the herd effect in the same model used in the random regression following an iterative approach (Gibbs sampling). In a simulation study, this approach gave higher estimates of the variance of the linear random regression term than the traditional approach, thus indicating more $\mathrm{G} \times \mathrm{E}$ (Su et al., 2006). The downside of the new approach is that it is more time consuming. It might, however, be interesting to compare the estimates from our approximate method

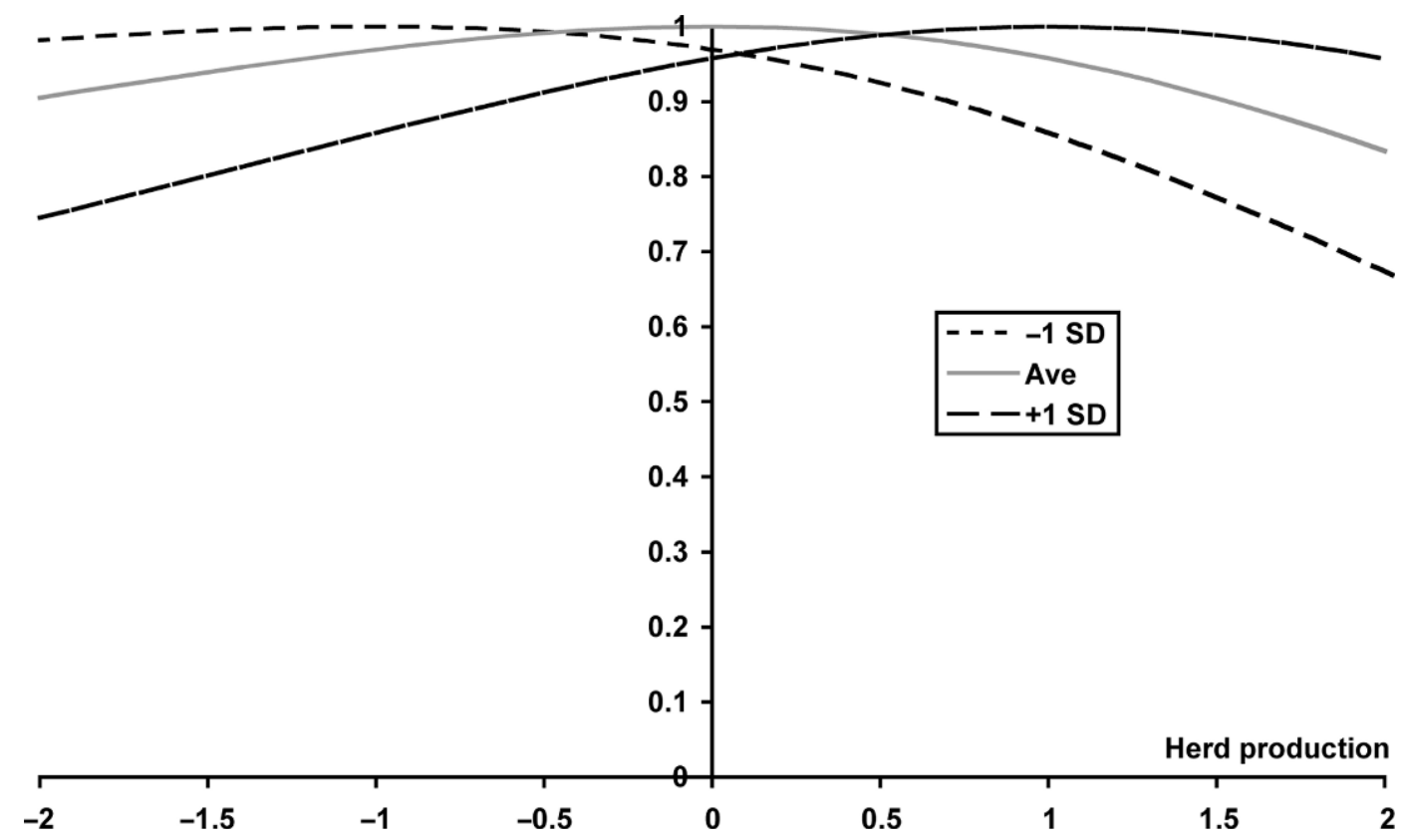

Figure 4. Genetic correlations for days to first service (DFS) across various herd production values. Graphs shown are between herds with environmental values of -1 , average, and $+1 \mathrm{SD}$, respectively, and all other environmental values. 


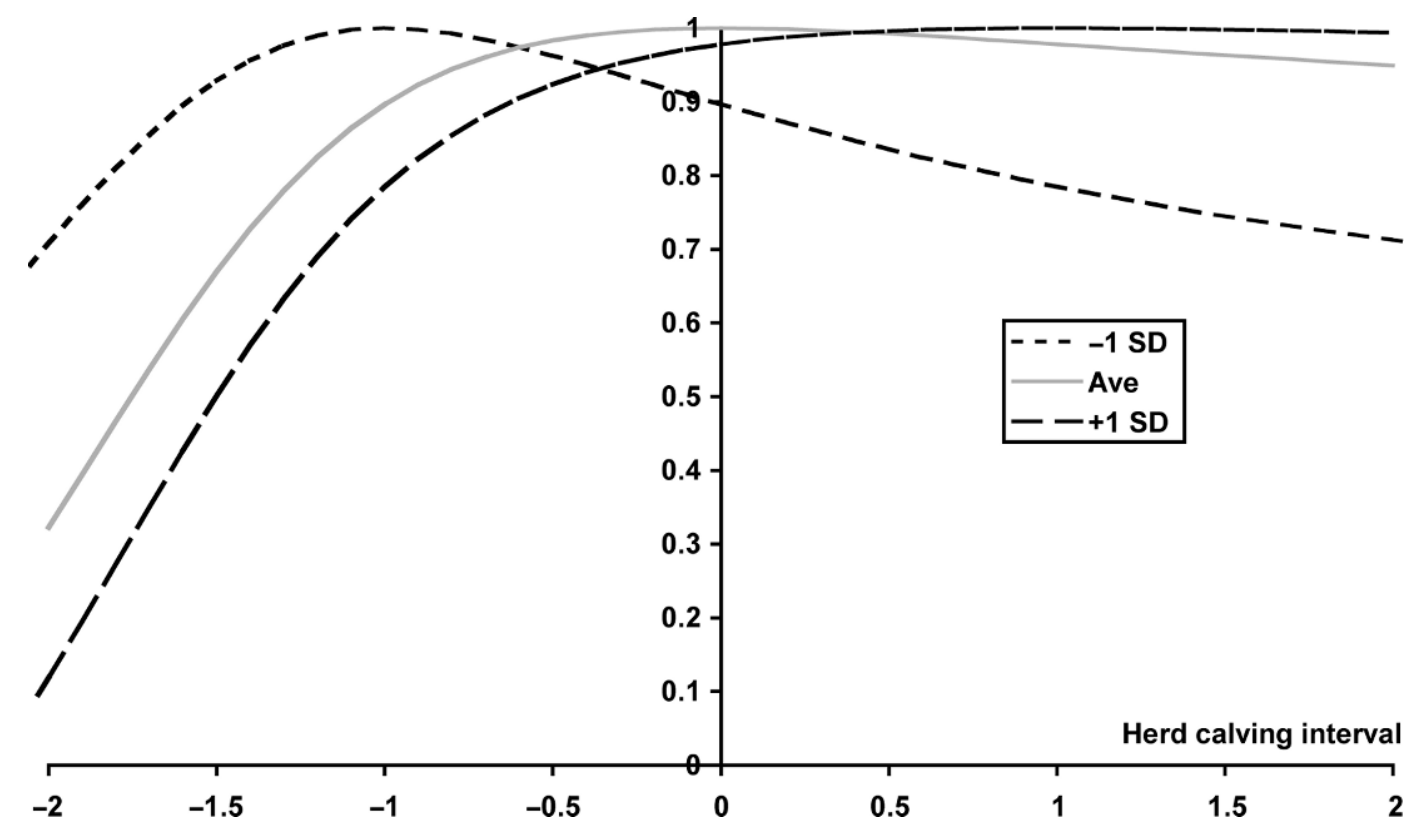

Figure 5. Genetic correlations for calving interval (CI) across various herd CI values. Graphs shown are between herds with environmental values of $-1 \mathrm{SD}$, average, and $+1 \mathrm{SD}$, respectively, and all other environmental values.

with those from the method of $\mathrm{Su}$ et al. (2006) using real data.

\section{Results from the MT Analysis}

Estimates of variance components, heritabilities, and genetic correlations across environments from the MT analysis of DFS and CI are shown in Table 6. In the same table, values for genetic correlations calculated from the RN models are shown, based on the average environmental value for the 2 quartiles (Table 3 ).

For NR56 and NINS, all multiple trait correlations were close to unity or not significantly different from unity, with 2 exceptions: for NR56 with respect to

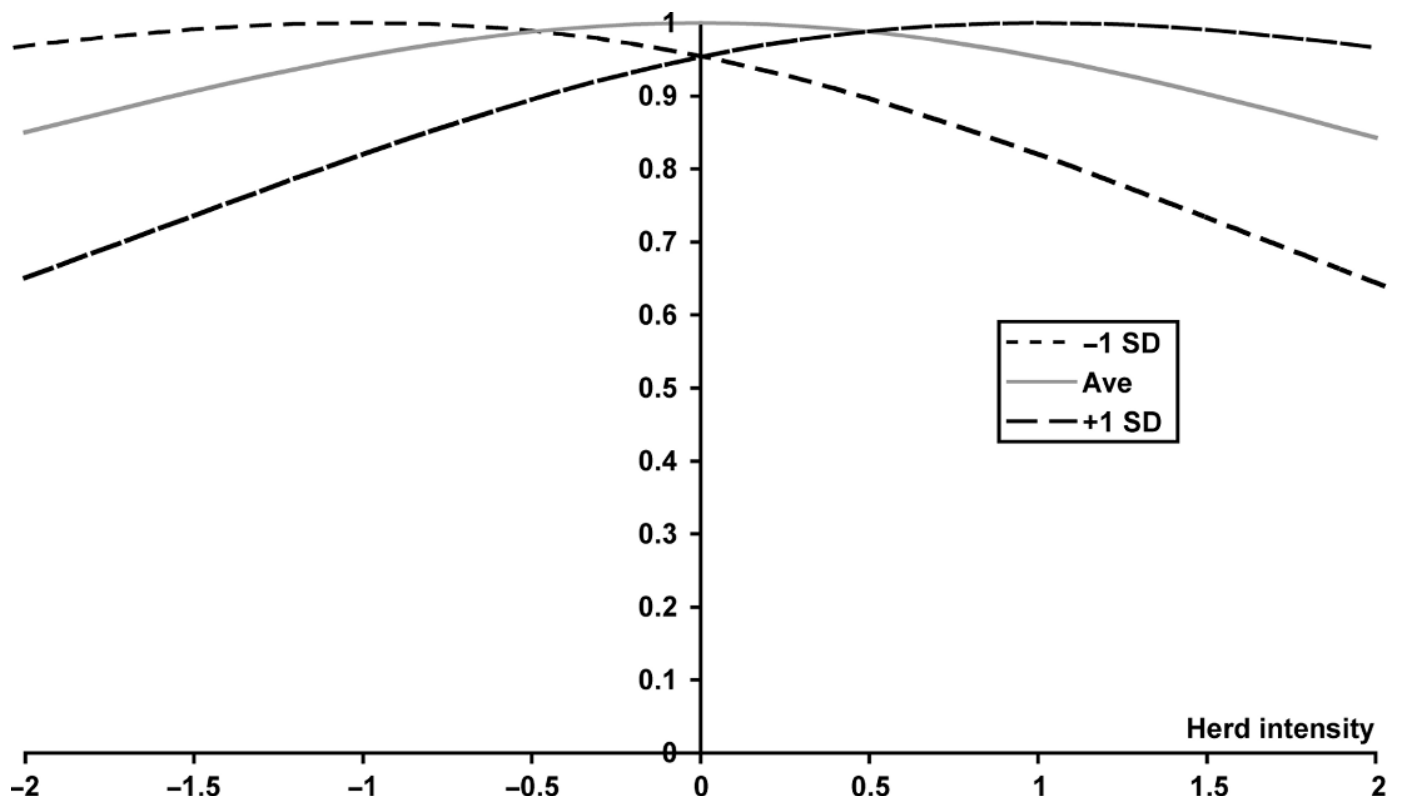

Figure 6. Genetic correlations for calving interval (CI) across various herd intensity values. Graphs shown are between herds with environmental values of -1 , average, and $+1 \mathrm{SD}$, respectively, and all other environmental values. 
production and intensity, correlations were $0.58 \pm 0.29$ (nonsignificant) and $0.27 \pm 0.27$. However, there was so little genetic variation in this trait (Table 5), especially for the high quartile (Figure 2), that we caution against interpreting these correlations as an indication of $\mathrm{G} \times \mathrm{E}$. Also, there was no indication of $\mathrm{G} \times \mathrm{E}$ from the $\mathrm{RN}$ model for these traits.

For DFS with respect to production and intensity environments, the estimates of genetic correlations between the 2 environments were around 0.8 , which corresponded well with estimates from the RN models. However, with respect to the environmental scale DFS, the MT analysis gave a correlation of unity, whereas the RN model indicated a correlation of about 0.8 . The only MT correlation significantly different from unity was that of 0.8 between low- and high-intensity herds.

For CI, the MT and RN genetic correlations were generally similar. Only 1 MT correlation (that with respect to herd production level) was significantly different from unity.

\section{General Discussion}

Considering all the evidence, it seems that $\mathrm{G} \times \mathrm{E}$ exists for DFS and CI with respect to herd-average production or intensity, genetic correlations being around 0.8 or slightly lower. The case for $\mathrm{G} \times \mathrm{E}$ with respect to the fertility environments is less convincing, mainly because it only appears in the RN model and only when the same trait is used as the environmental descriptor. On the other hand, there seems to be no $\mathrm{G} \times \mathrm{E}$ for NR56 and NINS.

As mentioned in the introduction, there are few studies of $\mathrm{G} \times \mathrm{E}$ for female fertility with which to make comparisons. Kolmodin et al. (2002) found high genetic correlations for days open between various environments graded on herd-average protein yield or days open.

Two studies have focused on grazing as the environmental descriptor. Boettcher et al. (2003) found a low correlation of 0.64 (SE 0.27) for CI between herds with intensive grazing and conventional herds in Canada, but this correlation was not significantly different from unity. Only 22 grazing herds were included, and fewer than 4,000 fertility records were available from these herds. They also found almost twice the heritability for CI in grazing herds (5.2\%). Kearney et al. (2004) found no $\mathrm{G} \times \mathrm{E}$ for DFS and number of services per conception between grazing herds (most forage from grass for at least 6 mo per year) and control herds (with little or no grazing) in the United States for days open. Kearney et al. (2004) did find a significant genetic correlation of 0.74 (SE 0.12) for days open in one subset of data but not in the other. In our study we did not specifically distinguish between herds with mainly spring calving
Table 6. Estimates of genetic correlations $\left(r_{\mathrm{g}}\right)$ from the multiple-trait (MT) analysis treating trait values in low and high quartiles for the environmental descriptors as separate traits (SE in subscript) and from the reaction norm (RN) model [4] between the average environmental values in the low and high environments ${ }^{1}$

\begin{tabular}{lll}
\hline Trait and environmental descriptor $^{2}$ & Model & \multicolumn{1}{c}{$r_{\mathrm{g}}$} \\
\hline DFS & & \\
DFS & MT & $1.00 \dagger_{0.12}$ \\
& RN & $\mathbf{0 . 7 8}$ \\
CI & MT & $1.00 \dagger_{0.11}$ \\
& RN & $1.00 \dagger^{\circ}$ \\
NR56 & MT & $0.94_{0.11}$ \\
& RN & 0.90 \\
Production index & MT & $0.84_{0.11}$ \\
Intensity index & RN & $\mathbf{0 . 7 7}$ \\
& MT & $\mathbf{0 . 8 0} 0.11$ \\
CI & RN & $\mathbf{0 . 8 4}$ \\
DFS & & \\
& MT & $1.00 \dagger_{0.12}$ \\
CI & RN & 0.98 \\
NR56 & MT & $0.88_{0.11}$ \\
Production index & RN & $\mathbf{0 . 7 2}$ \\
Intensity index & MT & $0.91_{0.12}$ \\
& RN & 0.92 \\
& MT & $\mathbf{0 . 7 4}$ \\
\hline
\end{tabular}

${ }^{1}$ Bold figures indicate models with statistically significant slope parameters or genetic correlations from the multiple trait analysis significantly different from unity (one-sided $5 \%$ test).

${ }^{2} \mathrm{DFS}=$ days to first service; $\mathrm{CI}=$ calving interval; NR56 = nonreturn rate at $56 \mathrm{~d}$.

$\dagger$ Genetic correlation $>0.995$, rounded to 1.00 .

and year-round calving. However, as shown in Haskell et al. (2007), low-intensity (and low-production) herds were also predominantly spring calving and had a longer grazing period. This could therefore be part of the explanation for the $\mathrm{G} \times \mathrm{E}$ found with respect to these 2 environmental scales.

Calus et al. (2005) generally found strong genetic correlations between fertility expressed in different environments: the main exception was for conception to first service when the environment was herd-average body condition score. Windig et al. (2006) found a genetic correlation of around 0.9 between extreme fertility environments for DFS and NINS, but neither were significantly different from unity. The correlations with respect to production intensity were 0.91 to 0.97 , also nonsignificant.

The genetic correlations between environments found in our study were, in general, lower than those found in the literature. One possible way to respond to these results would be to estimate and present customized breeding values for, say, low- versus average- or high- production or intensity herds, or spring versus year-round calving herds. To what extent breeders and farmers would be willing to use these breeding values customized to specific herd types is still unknown. 
The environmental descriptor called intensity was not better than average production for identifying $\mathrm{G} \times \mathrm{E}$ for fertility. However, the intensity index was shown to be a good descriptor for analyzing $\mathrm{G} \times \mathrm{E}$ for longevity in $\mathrm{UK}$ data (Haskell et al., 2007) and if used for that purpose, it could be used for $\mathrm{G} \times \mathrm{E}$ for $\mathrm{DFS}$ and NINS.

\section{CONCLUSIONS}

There was some $\mathrm{G} \times \mathrm{E}$ detected for female fertility traits with respect to herd-average production or intensity, but it is unclear whether its size is large enough for farmers to use customized breeding values of bulls within the United Kingdom, if such breeding values were available. However, given the limited range of environments studied, there would be merit in continuing this line of investigation across more extreme environments, for example, by pooling data from different countries.

\section{ACKNOWLEDGMENTS}

The authors thank Ian White (University of Edinburgh) for statistical advice and Raphael Mrode (Scottish Agricultural College) for extracting the data from the Scottish Agricultural College database.

\section{REFERENCES}

Boettcher, P. J., J. Fatehi, and M. M. Schutz. 2003. Genotype $\times$ environment interactions in conventional versus pasture-based dairies in Canada. J. Dairy Sci. 86:383-389.

Calus, M. P., J. J. Windig, and R. F. Veerkamp. 2005. Associations among descriptors of herd management and phenotypic and genetic levels of health and fertility. J. Dairy Sci. 88:2178-2189.

Haskell, M. J., S. Brotherstone, and I. White. 2007. Characterization of dairy farm environments in Great Britain, and the effect of farm environment on cow lifespan. J. Dairy Sci. 90:5316-5323.

Kearney, J. F., M. M. Schutz, and P. J. Boettcher. 2004. Genotype $\times$ environment interaction for grazing vs. confinement. II. Health and reproductive traits. J. Dairy Sci. 87:510-516.

Kolmodin, R., E. Strandberg, P. Madsen, J. Jensen, and H. Jorjani. 2002. Genotype by environment interaction in Nordic dairy cattle studied by use of reaction norms. Acta Agric. Scand. A Anim. 52:11-24.

Madsen, P., and J. Jensen. 2000. A user's guide to DMU-A package for analysing multivariate mixed models. Danish Inst. Agric. Sci. (DIAS), Dept. Animal Breeding and Genetics, Research Centre Foulum, Tjele, Denmark.

Mantysaari, E. A., R. L. Quaas, and Y. T. Grohn. 1991. Simulation study on covariance component estimation for 2 binary traits in an underlying continuous scale. J. Dairy Sci. 74:580-591.

Su, G., P. Madsen, M. S. Lund, D. Sorensen, I. R. Korsgaard, and J. Jensen. 2006. Bayesian analysis of the linear reaction norm model with unknown covariates. J. Anim. Sci. 84:1651-1657.

Windig, J. J., M. Calus, B. Beerda, and R. F. Veerkamp. 2006. Genetic correlations between milk production and health and fertility depending on herd environment. J. Dairy Sci. 89:1765-1775. 